\title{
A Rare Case of Autoimmune Gastritis and Pernicious Anemia Associated with Vitiligo
}

Sabarinathan Ramanathan, Premkumar Karunakaran, Kani Shaik Mohamed, Ratnakar Kini, Pugazhendhi Thangavel, Murali Ananthavadivelu

Institute of Medical Gastroenterology, Madras Medical College and Rajiv Gandhi Government General Hospital, Chennai, Tamil Nadu, India.

Corresponding Author:

Dr. Sabarinathan Ramanathan

Email: gastrosabari@gmail.com

This is an Open Access article distributed under the terms of the Creative Commons Attribution License (creativecommons.org/ licenses/by/3.0).

Received : February 21, 2017

Accepted : April 24, 2017

Published : May 25, 2017

\begin{abstract}
Background: Autoimmune gastritis is a chronic inflammatory condition of gastric mucosa involving fundus and body of stomach. It presents with hypochlorhydria or achlorhydria and pernicious anemia secondary to anti-parietal cell antibody and anti-intrinsic factor antibody respectively. It most commonly occurs in older people. Case Report: Here we present a rare case of autoimmune gastritis and pernicious anemia associated with vitiligo in a young male patient. Conclusion: In evaluating pernicious anemia patient, search for co-existence of any autoimmune disorder even in younger population.
\end{abstract}

Keywords: Anemia, Gastritis, Glossitis, Helicobacter pylori, Vitiligo.

\section{Introduction}

Autoimmune gastritis (AIG) is a chronic inflammatory condition that presents with oxyntic mucosal atrophy of the gastric corpus and fundus [1]. AIG is characterized by an immunological dysregulation of the sensitized T-lymphocytes with production of autoantibodies causing destruction of parietal cell proton pump and intrinsic factor resulting in hypochlorhydria or achlorhydria and pernicious anemia respectively. The worldwide prevalence of the disease has been estimated as $2-5 \%$ [2]. Here, we present a young male patient with vitiligo who had pernicious anemia and on evaluation was found to have features suggestive of AIG.

\section{Case Report}

A 24-year-old male patient presented with a history of lethargy and easy fatigability for about four months. He had a history of multiple hypopigmented patches around the eyes, right chest, and left thigh with graying of scalp hairs in the occipital region of four years duration [Fig.1]. There was no significant family history or associated comorbidities. On general examination he was pale and had glossitis. Systemic examination was normal. Dermatologist opinion was sought for the well-demarcated hypo-pigmented patches and was diagnosed as vitiligo.

Laboratory data revealed a picture of pancytopenia with hemoglobin $(\mathrm{Hb}): 5.1 \mathrm{~g} / \mathrm{dL}$, platelet count: $1,10,000$ cells $/ \mathrm{mm}^{3}$, total leucocyte count: 3,500 cells $/ \mathrm{mm}^{3}$, packed cell volume (PCV): $25.3 \%$, and mean corpuscular volume (MCV): 118.4 fl. Serum ferritin level was $79.4 \mathrm{ng} /$ $\mathrm{mL}(30-400 \mathrm{ng} / \mathrm{mL})$. Fasting blood sugar, renal function test, and liver function tests were found to be normal. Peripheral blood smear showed erythropenia, macrocytic normochromic red blood cells, moderate aniso-poikilocytosis with good number of macro-ovalocytes, leucocytopenia and mild thrombocytopenia, features consistent with pancytopenia and macrocytic anemia [Fig.2]. Ultrasonography of abdomen was normal. Upper 
gastrointestinal endoscopy showed a shiny surface and pale gastric mucosa with an effacement of gastric rugal folds, along with prominent submucosal vessels in both body and fundus of the stomach, while the incisura, antrum of the stomach and duodenum upto second part were normal [Fig.3]. Rapid urease test for Helicobacter pylori was negative. Multiple separately collected biopsies were taken from gastric antrum, body and fundus.

Histo-pathological examination (HPE) of the tissue from body and fundus in scanner view showed bits of gastric mucosa with areas of atrophy in the form of reduction in the number of glands which were replaced by the sheets of chronic inflammatory cells and fibrosis. Only few spared crowded mucosal glands were seen at places. Adjacent fragment showed moderate diffuse chronic inflammatory infiltrate in the lamina propria, overall features suggestive of atrophic gastritis. High power view depicted lymphoid infiltrate forming lymphoid follicle at the focus along with diffuse infiltration causing glandular atrophy [Fig.4]. Giemsa stain for H. pylori was negative. With this background, anti-parietal cell antibody and anti-intrinsic factor antibody were done to seek for the diagnosis of AIG and both were found to be positive. Fasting gastrin level was 362 $\mathrm{pg} / \mathrm{mL}(13-115 \mathrm{pg} / \mathrm{mL})$. Serum ferritin level was $79.4 \mathrm{ng} / \mathrm{mL}(30-400 \mathrm{ng} / \mathrm{mL})$. Serum vitamin $B_{12}$ level was found to be $78.8 \mathrm{pg} / \mathrm{mL}(120-770 \mathrm{pg} /$ $\mathrm{mL})$. Thyroid function test was normal and antithyroid peroxidase antibody was also found to be negative. Thus, diagnosis of AIG with pernicious anemia was made. Treatment was initiated with intramuscular injection of $1000 \mu \mathrm{g}$ of vitamin $\mathrm{B}_{12}$ as per protocol. The protocol being $1000 \mu \mathrm{g}$ of vitamin $B_{12}$ given intramuscularly every week for four weeks to be followed by one dose every month for lifelong. Our patient responded well to the treatment and he was on regular follow up. He was also treated with topical $0.1 \%$ tacrolimus ointment and hydrocortisone cream given on alternate days at bedtime for vitiligo.

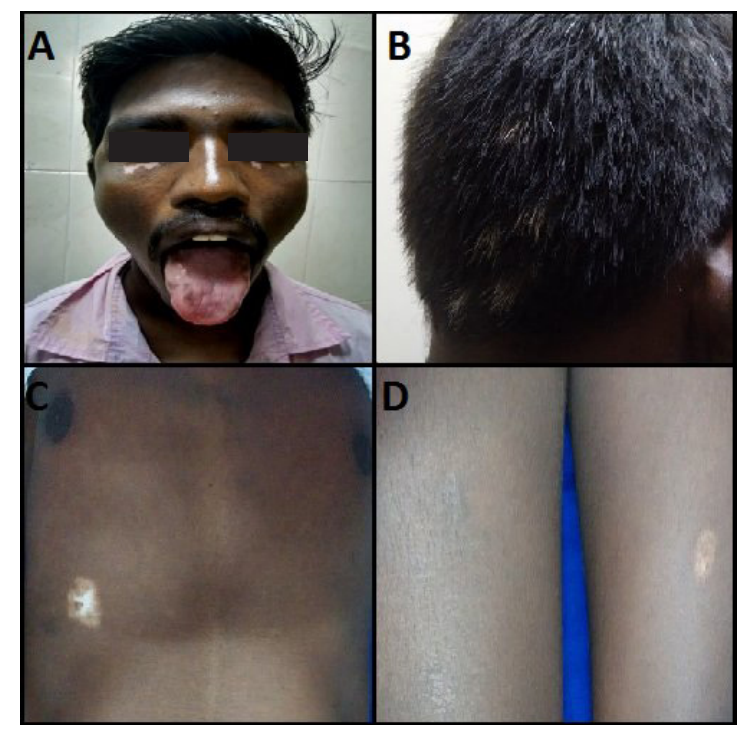

Fig.1(A): Vitiligo patches around the eyes. (B): Graying of scalp hairs in the occipital region. (C,D): Vitiligo patches in right chest and left thigh.

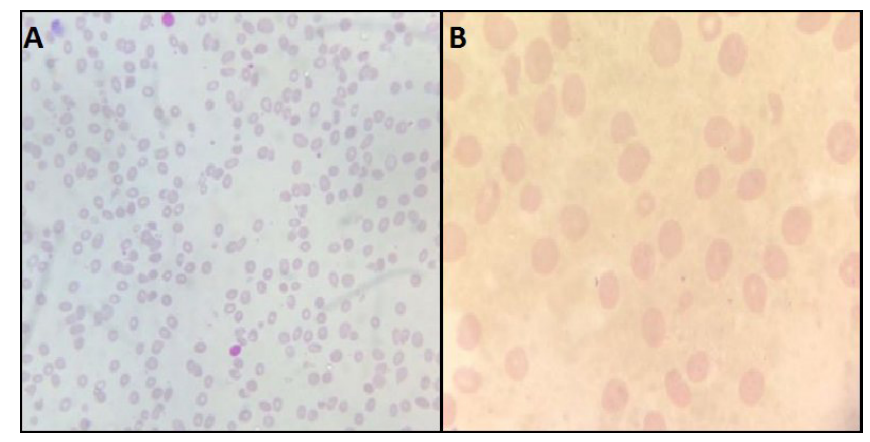

Fig.2(A): Peripheral smear picture shows macrocytic anemia, mild leucopenia and mild thrombocytopenia (Leishman stain, 10X). (B): Higher magnification showing many macroovalocytes (Leishman stain, 100X, oil immersion).

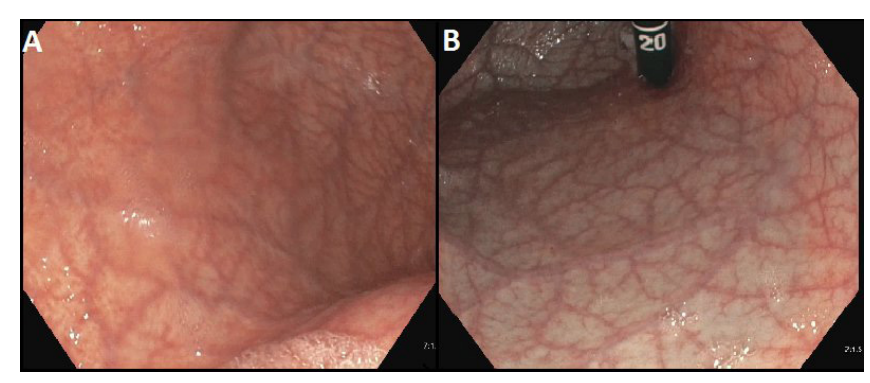

Fig.3(A): Upper gastrointestinal endoscopy shows a shiny surface and pale gastric mucosa with an effacement of gastric rugal folds, along with prominent submucosal vessels in both body and (B): fundus of the stomach. 


\section{Discussion}

Autoimmune gastritis occurs secondary to interaction between various host-related factors (genetic susceptibility) and environmental factors (H. pylori mediated) resulting in immunologically mediated sensitization of T-lymphocytes. Further, they usher the production of autoantibodies against parietal cell proton pumps and intrinsic factor that leads onto their destruction resulting in hypochlorhydria or achlorhydria and pernicious anemia respectively [2]. Though AIG and environmental metaplastic atrophic gastritis (secondary to $\mathrm{H}$. pylori infection) are separate entities, recent studies present the concordance of $H$. pylori infection with AIG in a significant proportion of patients [3]. Our patient had AIG without an overlapping $H$. pylori infection.

Pernicious anemia (PA), the macrocytic anemia also termed as Biermers disease or Addisonian anemia is an autoimmune disorder. It presents as vitamin $\mathrm{B}_{12}$ (cobalamin) deficiency, secondary to the deficiency of intrinsic factor, which is responsible for avid binding and promoting the transport of dietary cobalamin [4]. Usually pernicious anemia occurs in advanced stage of AIG. Both AIG and pernicious anemia are viewed as a disease of elderly female and common in Northern European and African American descent but very rare in Indian population [5]. The prevalence of PA in young $<30$ years is $4 \%$ [4]. AIG and pernicious anemia often co-exist with other autoimmune diseases such as autoimmune thyroiditis and type-1 diabetes mellitus. Though in few literatures, association of AIG and/or pernicious anemia with vitiligo, an autoimmune disorder presenting as depigmented macules or patches of skin is reported, it is regarded as an extremely rare entity accounting for only $2-8 \%$ [3]. Our patient is a young ( $<30$ years) male of Indian origin who presented with pernicious anemia due to AIG (no overlapping of $H$. pylori) associated with an autoimmune disorder vitiligo. The clinical presentation of PA is insidious and the commonly

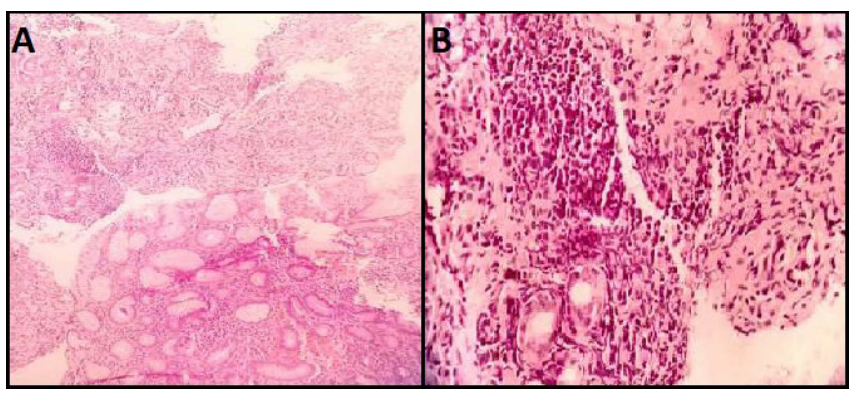

Fig.4(A): Glandular destruction by lymphocytic infiltrate and fibrosis (H\&E stain, 4X). (B): Lymphocytic infiltrate with reactive follicle and severe glandular atrophy $(H \& E$ stain, $40 X)$.

reported hematological manifestations include neutropenia, thrombocytopenia or pancytopenia [4]. He also presented with non-specific symptoms and had pancytopenia. Endoscopy is regarded as the gold standard investigation for diagnosis of AIG [1]. He was promptly evaluated and treated with intramuscular injection of vitamin $\mathrm{B}_{12}$ and he also responded well to the therapy. AIG is deemed as a risk factor for the development of gastric carcinoma and carcinoids while presence of PA increases the risk further [3]. Hence, was advised to undergo endoscopic surveillance with biopsy every 3 years as per European MAPS (Management of Precancerous Conditions and Lesions in the Stomach) guidelines [6].

\section{Conclusion}

As AIG and PA are often under-diagnosed entities, while evaluating a patient with PA it is mandatory to search for co-existence of any autoimmune disorder even in younger population. A prompt evaluation of the patient using endoscopy and biopsies of mucosa should be done to rule out AIG, thereby it could help in early diagnosis and treatment of gastric carcinoma and carcinoids.

Contributors: SR, PK: Manuscript writing, case management; KSM, RK: manuscript revision, case management; PT, MA: critical review of manuscript for intellectual content. SR will act as guarantor. All authors approved final version of the manuscript.

Funding: None; Competing interests: None stated. 


\section{References}

1. Kulnigg-Dabsch S. Autoimmune gastritis. Wiener Medizinische Wochenschrift. 2016;166:424-430.

2. Coati I, Fassan M, Farinati F, Graham DY, Genta RM, Rugge M. Autoimmune gastritis: Pathologist's viewpoint. World J Gastroenterol. 2015;21:1217912189.

3. Park JY, Lam-Himlin D, Vemulapalli R. Review of autoimmune metaplastic atrophic gastritis. Gastrointest
Endosc. 2013;77:284-292.

4. Behera P, Tripathy K, Panigrahi R, Tripathy S. Pernicious anemia in young: A case report with review of literature. Int J Med Res Heal Sci. 2015;4:902.

5. Basha SJ, Shetty NR, Devarbhavi H. Pernicious Anaemia with Gastric Carcinoids. J Assoc Physicians India. 2016;64:2015-2016.

6. Dinis-Ribeiro M, Areira M, A.C. de Vries D. Managment of precancerous conditions and lesiond in the stomach (MAPS). Endoscopy. 2012;44:74-94. 